\title{
MicroRNA-375 inhibits oral squamous cell carcinoma cell migration and invasion by targeting platelet-derived growth factor-A
}

\author{
ZHENG-HAO CAO* , JING-LIANG CHENG ${ }^{*}$, YONG ZHANG, CHUN-XIAO BO and YAN-LIANG LI \\ Department of MRI, The First Affiliated Hospital of Zhengzhou University, Zhengzhou, Henan 450052, P.R. China
}

Received November 10, 2015; Accepted November 15, 2016

DOI: $10.3892 / \mathrm{mmr} .2016 .6057$

\begin{abstract}
MicroRNA-375 (miR-375) serves an important role in cancer development and growth. However, little is known about the role of miR-375 in the regulation of oral squamous cell carcinoma (OSCC) metastasis and invasion. The present study measured the expression levels of miR-375 in Tca8113, UM2, UM1 and CAL-27 cell lines, using reverse transcription-quantitative polymerase chain reaction. The results demonstrated that miR-375 expression levels were significantly reduced in UM1 and CAL-27 (highly metastatic) compared with Tca8113 and UM2 (less aggressive) OSCC cell lines. Furthermore, it was revealed that overexpression of miR-375 suppressed the migration and invasion of UM1 cells. Based on a luciferase reporter assay, platelet-derived growth factor-A (PDGF-A) was identified as a direct target gene of miR-375. Additionally, overexpression of PDGF-A significantly reversed the effect of miR-375 on cell migration and invasion in UM1 cells. These data demonstrated that miR-375 suppressed OSCC cell migration and invasion by targeting PDGF-A, which may be a potential therapeutic target for the treatment of OSCC.
\end{abstract}

\section{Introduction}

Oral squamous cell cancer (OSCC), characterized by the development of malignant tumors in the mouth, is the eighth most common type of cancer worldwide (1). In China, the incidence of oral cancer is $\sim 3-6 / 100,000$ individuals; however, this number is increasing every year. Smoking, alcohol abuse and betel quid-chewing serve important roles in the etiology of oral cancer. At present, although have been certain advances

Correspondence to: Dr Yong Zhang, Department of MRI, The First Affiliated Hospital of Zhengzhou University, 1 Jianshe Road, Zhengzhou, Henan 450052, P.R. China

E-mail: 15838128890@139.com

*Contributed equally

Key words: oral squamous cell carcinoma, microRNA-375, migration, invasion, platelet-derived growth factor-A in surgery, radiotherapy and chemotherapy for oral cancer, the mortality rate remains high (2). Tumor metastasis is the primary cause of mortality in patients with oral cancer (3). Therefore, an increased understanding of the mechanisms underlying oral cancer metastasis is required to develop novel therapeutic strategies.

microRNAs (miRNAs) suppress gene expression levels via direct binding to the $3^{\prime}$ untranslated region (UTR) of target mRNA, thereby regulating the expression levels of various oncogenes and tumor suppressors involved in metastasis and tumor progression (4-6). miRNA (miR)-375 may function as a tumor suppressor, and there is increasing evidence to suggest that it may be useful as a diagnostic biomarker and a therapeutic target for cancer treatment. Winther et al (7) demonstrated that miR-375 served as a prognostic biomarker in patients with primary esophageal squamous cell carcinomas. In laryngeal squamous cell carcinoma, the miR-21/miR-375 ratio was revealed as an independent prognostic marker (8). In colorectal carcinoma, Mao et al (9) demonstrated that miR-375 was a potential therapeutic target for the treatment of colorectal cancer, which reduces the proliferation of conditionally reprogrammed cells via regulating the expression levels of the Kruppel-like factor (KLF)4 protein. In tongue squamous cell carcinoma, overexpression of miR-375 inhibited specificity protein 1 expression levels, which resulted in subsequent downregulation of cyclin D1. The same study suggested that miR-375 may inhibit cell growth in tongue squamous cell carcinoma (10). Song et al (11) revealed that miR-375 modulated the radiosensitivity of high-risk human papilloma virus-positive cervical cancer cells by targeting ubiquitin-protein ligase E3A via the tumor suppressor p53 signaling pathway. In OSCC, although miR-375 was demonstrated to be a potentially significant early-stage biomarker and therapeutic target (12), its exact functions and underlying mechanisms associated with cancer metastasis and invasion remain unclear.

The present study investigated the role of miR-375 in the regulation of OSCC cell migration and invasion. These data demonstrated that miR-375 was downregulated in highly metastatic OSCC cell lines, and that its overexpression suppressed OSCC cell migration and invasion. Mechanistic studies revealed that miR-375 may directly bind to the 3'-UTR of platelet-derived growth factor subunit A (PDGF-A) and downregulate its expression levels, which may suppress OSCC 
cell migration and invasion. The present study provides important insight into miR-375-mediated regulation of OSCC cell migration and metastasis.

\section{Materials and methods}

Cell lines and culture. The CAL-27, Tca8113, UM1 and UM2 OSCC cell lines (China Center for Type Culture Collection, Wuhan, China) were cultured in Dulbecco's modified Eagle's medium (DMEM) supplemented with $10 \%$ fetal bovine serum (FBS), $100 \mathrm{U} / \mathrm{ml}$ penicillin and $100 \mu \mathrm{g} / \mathrm{ml}$ streptomycin (Gibco; Thermo Fisher Scientific, Inc., Waltham, MA, USA). All cells were incubated at $37^{\circ} \mathrm{C}$ in $5 \% \mathrm{CO}_{2}$.

Transfection with miR-375 mimic and PDGF-A. miR-negative control (miR-NC, 5'-UCACAACCUCCUAGA AAGAGU AGA-3') and miR-375 mimic (5'-UUUGUUCGUUCGGCU CGCGUGA-3') were purchased from GenePharma Co., Ltd. (Shanghai, China). The full-length PDGF-A (NM_002607.5) was cloned and inserted into the pcDNA3.1 expression plasmid (Promega Corporation, Madison, WI, USA). Cells were plated at $50 \%$ confluence and transfected with $300 \mathrm{nM}$ miR-NC, miR-375 mimic and/or $10 \mu \mathrm{M}$ PDGF-A or pcDNA3.1 empty vector using the Lipofectamine ${ }^{\circledR} 2000$ reagent (Invitrogen; Thermo Fisher Scientific, Inc.), according to the manufacturer's protocol. Cells were harvested 24 or $48 \mathrm{~h}$ after transfection and subjected to further analysis.

RNA extraction and reverse transcription-quantitative polymerase chain reaction $(R T-q P C R)$ analysis. Total RNA was extracted from cultured cells using TRIzol ${ }^{\circledR}$ reagent (Invitrogen; Thermo Fisher Scientific, Inc.) according to the manufacturer's protocol. miR-375 expression levels were analyzed using the TaqMan Advanced miRNA assay (Applied Biosystems; Thermo Fisher Scientific, Inc.) and U6 served as an internal control. To evaluate PDGF-A mRNA expression levels, RT-qPCR was performed using the PrimeScript RT Reagent kit and SYBR ${ }^{\circledR}$ Premix Ex Taq reagent (Takara Biotechnology Co., Ltd., Dalian, China) according to the manufacturer's protocol. GAPDH served as an internal control. RT-qPCR was performed on an Applied Biosystems 7500 system (Applied Biosystems; Thermo Fisher Scientific, Inc.). The PDGF-A and GAPDH primer sequences used for RT-qPCR are presented in Table I. Gene expression levels were measured in triplicate, quantified using the $2^{-\Delta \Delta \mathrm{Cq}}$ method, and normalized to the control (13). The qPCR was conducted using the following conditions: $95^{\circ} \mathrm{C}$ for $5 \mathrm{~min}$ followed by 40 cycles of $95^{\circ} \mathrm{C}$ for $15 \mathrm{sec}$ and $60^{\circ} \mathrm{C}$ for $60 \mathrm{sec}$.

Transwell migration and invasion assays. Cell migration and invasion were assessed using a Transwell migration assay. For this, UM1 cells were harvested, and $5 \times 10^{4}$ cells in $200 \mu \mathrm{l}$ DMEM containing $0.1 \%$ FBS were placed into the upper chamber of an insert ( $8-\mu \mathrm{m}$ pore size; BD Biosciences, San Jose, CA, USA). The lower chamber was filled with $600 \mu 1$ DMEM containing 10\% FBS. Migrating cells were imaged using a Leica light microscope (Leica Microsystems $\mathrm{GmbH}$, Wetzlar, Germany; magnification, x200) in five randomly selected fields per well, and the mean count was calculated. For the invasion assays, $5 \times 10^{4}$ cells were seeded into the upper chamber, which had been precoated with Matrigel (BD Biosciences) and incubated for $24 \mathrm{~h}$. Following the removal of cells from the upper chamber with a cotton swab, the cells in the lower chamber were fixed with $4 \%$ paraformaldehyde, stained with $0.1 \%$ crystal violet solution in $20 \%$ ethanol, and counted in five randomly-selected fields, using a phase contrast light microscope at a magnification x200 (Olympus Corporation, Tokyo, Japan), following which the mean count was calculated. The assays were performed in triplicate.

Western blotting. The different experimental groups of UM1 cells were harvested and lysed using radioimmunoprecipitation assay buffer (Takara Biotechnology Co., Ltd.) and the total protein concentration was determined using the Bicinchoninic Acid Protein Assay kit (Beyotime Institute of Biotechnology, Nantong, China). Proteins $(30 \mu \mathrm{g})$ were separated by $8 \%$ SDS-PAGE and transferred onto polyvinylidene difluoride membranes. After blocked with $5 \%$ non-fat milk in Tris-buffered saline containing $0.05 \%$ Tween-20 (TBST) for $1 \mathrm{~h}$ at $37^{\circ} \mathrm{C}$, the membranes were incubated with a polyclonal rabbit anti-human PDGF-A antibody (1:500; cat. no. ab125268; Abcam, Cambridge, MA, USA) or a monoclonal rabbit anti-human $\beta$-actin antibody $(1: 2,000$; cat. no. ab115777; Abcam) for $1 \mathrm{~h}$ at $37^{\circ} \mathrm{C}$. Membranes were incubated with a horseradish peroxidase-conjugated goat anti-rabbit IgG H\&L secondary antibody (1:10,000; cat. no. ab97080; Abcam) for $40 \mathrm{~min}$ and proteins were visualized using an Enhanced Chemiluminescence reagent (Pierce; Thermo Fisher Scientific, Inc.). The expression levels of the proteins of interest were normalized against the expression levels of $\beta$-actin. Quantification was performed using ImageJ version 6.0 (National Institutes of Health, Bethesda, MD, USA).

Reporter vector construction and luciferase reporter assay. miR-375 targets were predicted using the online miRNA target prediction software TargetScan (www.targetscan.org) and miRanda (www.microrna.org/microrna/home.do). To determine whether the 3'-UTR of PDGF-A mRNA was a direct target of miR-375, the full-length wild-type 3'-UTR of PDGF-A and mutant 3'-UTR of PDGF-A were amplified and cloned into the psi-CHECK-2 vector (Promega Corporation, Madison, WI, USA). The primer sequences used for the construction of the reporter vector are presented in Table I. UM2 cells were co-transfected with $100 \mathrm{ng}$ plasmid and $200 \mathrm{nmol} / 1 \mathrm{miR}-375 \mathrm{mimic}$ or miR-NC in 24-well plates. Cell lysates were harvested $48 \mathrm{~h}$ after transfection. The firefly and Renilla luciferase fluorescence intensities were measured using the Dual-Luciferase Reporter assay system (Promega Corporation) and experiments were performed in triplicate.

Statistical analysis. All statistical analyses were performed using SPSS software version 19.0 (IBM SPSS, Armonk, NY, USA). Data are presented as the mean \pm standard deviation. A one-way analysis of variance was used to compare the differences between miR-375 expression levels in different OSCC lines and followed by a least significant difference post hoc test. A Student's t-test was used to compare the differences prior to and following treatment. $\mathrm{P}<0.05$ was considered to indicate a statistically significant difference. 
Table I. Primer sequences.

\begin{tabular}{ll}
\hline Primer name & \multicolumn{1}{c}{ Sequence (5'-3') } \\
\hline PDGF-A-F & CCCCTGCCCATTCGGAGGAAGAG \\
PDGF-A-R & TTGGCCACCTTGACGCTGCGGTG \\
GAPDH-F & ACACCCACTCCTCCACCTTT \\
GAPDH-R & TTACTCCTTGGAGGCCATGT \\
psiCHECK2-XhoI-F & CCGctcgagcgGAGGAAGAG \\
& AAGCATCGAG \\
psiCHECK2-NotI-R & ATAAGAATgcggccgcTAAGGCTCTC \\
& AGGAAGGTTTCTGTA \\
psiCHECK2-mut-F & TGTGTCCGAGAACACTCGGGATC \\
& GTTCGGAGACAGTGCACATTTGT \\
& TTAATGT \\
psiCHECK2-mut-R & ACATTAAACAAATGTGCACTGTC \\
& TCCGAACGATCCCGAGTGTTCTC \\
& GGACACA \\
\hline
\end{tabular}

F, forward; R, reverse; PDGF-A, platelet-derived growth factor subunit $\mathrm{A}$; mut, mutant. Lowercase letters represent the restriction enzyme sites. Bold letters represent the mutation site.

\section{Results}

miR-375 is downregulated in highly metastatic OSCC cell lines. To assess the role of miR-375 in the regulation of OSCC cell migration and invasion, miR-375 expression levels in UM2 and Tca8113 (less metastatic) and UM1 and CAL-27 (highly metastatic) OSCC cell lines were determined using RT-qPCR. The results demonstrated that expression levels of miR-375 were significantly decreased in UM1 compared with Tca8113 $(\mathrm{P}<0.001)$ and $\mathrm{UM} 2$ cell lines $(\mathrm{P}=0.003)$; however, no significant differences were identified in the CAL-27 cell line $(\mathrm{P}=0.278$; Fig. 1). The expression levels of miR-375 were reduced in the highly metastatic OSCC cell lines, particularly in UM1, compared with the less metastatic ones. Based on these results, the UM1 cell line was selected for further experiments.

Overexpression of miR-375 suppresses migration and invasion of UM1 cells. To assess the role of miR-375 in the regulation of OSCC cell migration and invasion, an miR-375 mimic or an miR-NC was transfected into UM1 cells. After $48 \mathrm{~h}$ of culture, UM1 cells were harvested for RT-qPCR. The results demonstrated that transfection of UM1 cells with an miR-375 mimic significantly increased miR-375 expression levels in $\mathrm{UM} 1$ cells $(\mathrm{P}=0.005)$ (Fig. 2A). Furthermore, following the transfection of cells with the miR-375 mimic or miR-NC for $48 \mathrm{~h}$, Transwell assays were performed to measure UM1 cell migration and invasion. The Transwell migration assay revealed that the ability of the cells to migrate through the membrane into the lower chamber was significantly inhibited in the miR-375 mimic-transfected cells compared with miR-NC-transfected cells ( $\mathrm{P}=0.002$; Fig. 2B). The Transwell invasion assay demonstrated that the ability of the cells to pass through a Matrigel-coated membrane into the lower chamber

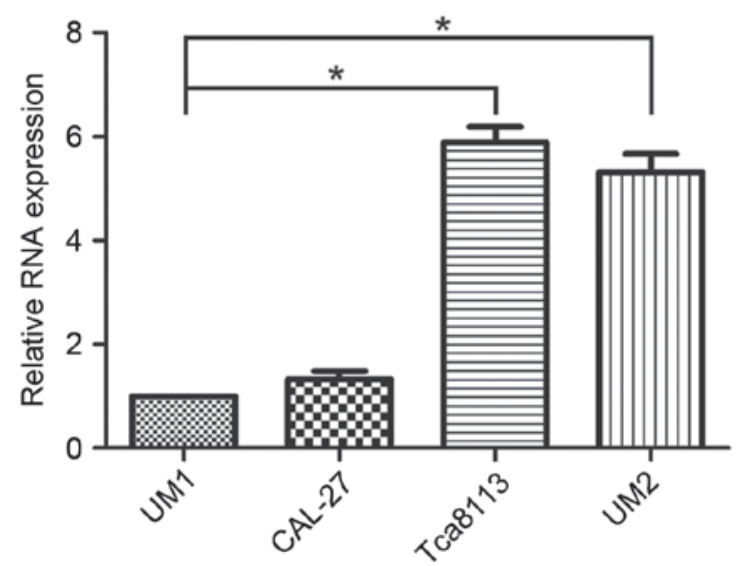

Figure 1. miR-375 is downregulated in highly metastatic OSCC cell lines. The miR-375 expression levels in UM1 and CAL-27 (highly metastatic) and UM2 and Tca8113 (less metastatic) OSCC cell lines were detected using reverse transcription-quantitative polymerase chain reaction. Data are presented as the mean \pm standard deviation, ${ }^{*} \mathrm{P}<0.05$. miR-375, microRNA-375; OSCC, oral squamous cell carcinoma.

was significantly decreased in the miR-375 mimic-transfected cells compared with the miR-NC-transfected cells $(\mathrm{P}<0.001$; Fig. 2C). Representative images of migrated and invaded cells are presented in Fig. 2D and E, respectively.

miR-375 regulates PDGF-A expression levels via targeting of its 3'-UTR. To further elucidate the mechanisms underlying miR-375-mediated suppression of UM1 cell migration and invasion, the targets of miR-375 were identified using the prediction algorithms of TargetScan and miRanda. This analysis identified PDGF-A as a potential target of miR-375, based on the putative binding site at positions 163-169 of the PDGF-A 3'-UTR (Fig. 3A). To demonstrate the association between miR-375 and PDGF-A, the protein expression levels of PDGF-A in the UM1, CAL-27, UM2 and Tca8113 cell lines were determined. The results confirmed that the UM1 and CAL-27 (highly metastatic) cell lines had greater levels of PDGF-A protein compared with the Tca8113 and UM2 (less metastatic) cell lines $(\mathrm{P}<0.001$, Tca8113 and UM2 compared with UM1; $\mathrm{P}=0.003, \mathrm{P}=0.009$, respectively; Tca8113 and UM2 compared with CAL-27; Fig. 3B and C). Luciferase reporter vectors, co-transfected into UM1 cells with miR-375 mimic or miR-NC, were used to further examine whether PDGF-A was the target of miR-375. These contained wild-type PDGF-A 3'-UTR or a mutant 3'UTR mutated at the predicted miR-375 binding site. A significant decrease in the luciferase activity of the reporter was observed for the wild-type PDGF-A 3'-UTR-containing vector compared with miR-NC $(\mathrm{P}<0.001)$, whereas this decrease did not occur when the target site was the mutated PDGF-A 3'-UTR ( $\mathrm{P}=0.158$; Fig. 3D). These results indicated that the sequence in the 163-169 bp region of the PDGF-A 3'-UTR interacted with miR-375, leading to the inhibition of PDGF-A expression levels in UM1 cells. Additionally, the effects of miR-375 overexpression on PDGF-A mRNA and protein levels were examined. As expected, overexpression of miR-375 did not cause degradation of PDGF-A mRNA (P=0.096) (Fig. 3E); however, it did result in a significant reduction in the protein expression levels 

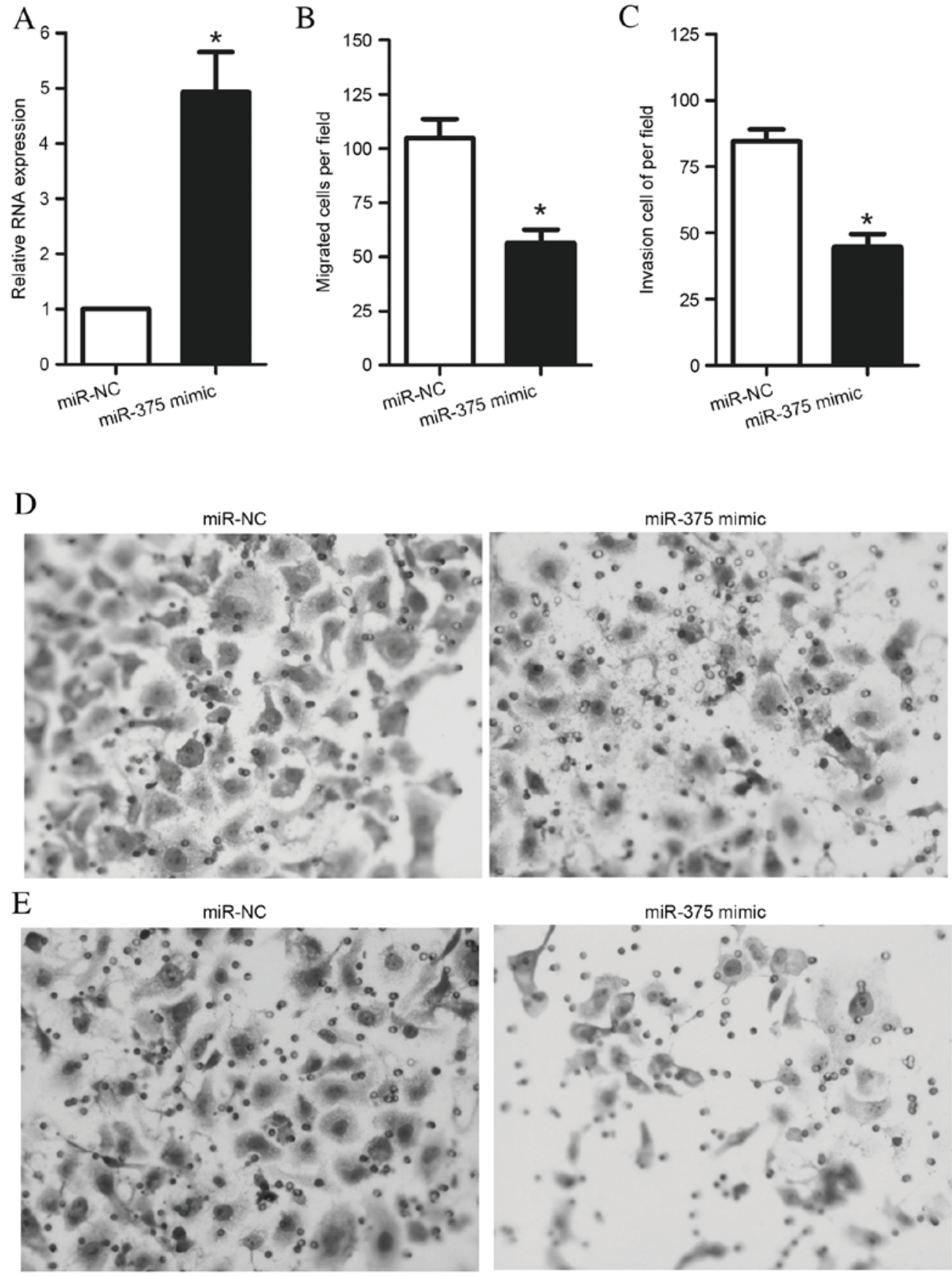

Figure 2. miR-375 overexpression suppresses the migration and invasion of UM1 cells. (A) The expression levels of miR-375 were detected using reverse transcription-quantitative polymerase chain reaction following transfection with a miR-375 mimic or miR-NC for $48 \mathrm{~h}$. The number of (B) migrating and (C) invading cells per field in the miR-NC and miR-375 mimic groups were calculated following Transwell assays. Representative images of UM1 cell (D) migration and (E) invasion. Data are presented as the mean \pm standard deviation. "P<0.05 vs. miR-NC. miR-375, microRNA-375; miR-NC, microRNA negative control.

of PDGF-A ( $\mathrm{P}=0.008$; Fig. $3 \mathrm{~F}$ and $\mathrm{G})$. These data indicated that miR-375 directly targeted the 3'-UTR of PDGF-A.

PDGF-A is involved in the miR-375-induced effects on migration and invasion of UM1 cells. To determine whether miR-375 contributes to UM1 migration and invasion via PDGF-A, UM1 cells were transfected with a PDGF-A expression vector for $48 \mathrm{~h}$. PDGF-A protein expression levels were increased in cells co-transfected with the PDGF-A expression vector and miR-375 mimic, compared with cells co-transfected with the $\mathrm{NC}$ vector and miR-375 mimic ( $\mathrm{P}<0.001$; Fig. $4 \mathrm{~A}$ and $\mathrm{B})$.
Transwell migration and invasion assays were performed, and representative images are presented in Fig. 4C and D, respectively. The Transwell migration assay demonstrated that the ability of these cells to migrate through the membrane into the lower chamber was significantly enhanced in miR-375 mimic- and PDGF-A-transfected cells compared with miR-375 mimic- and NC-transfected cells ( $\mathrm{P}=0.001$; Fig. 4E). Additionally, the Transwell invasion assays revealed that the ability of the cells to pass through the Matrigel-coated membrane into the lower chamber was significantly increased in miR-375 mimic- and PDGF-A-transfected cells compared 
A

hsa-miR-375 3' AGUGCGCUCGGCUUGCUUGUUU 5'

|I || || |

163-169 PDGFA 3' UTR wild 5' CGAGAACACUCGGGAGAACAAG 3' PDGFA 3' UTR mutant 5' CGAGAACACUCGGGAUCGUUCGG 3'
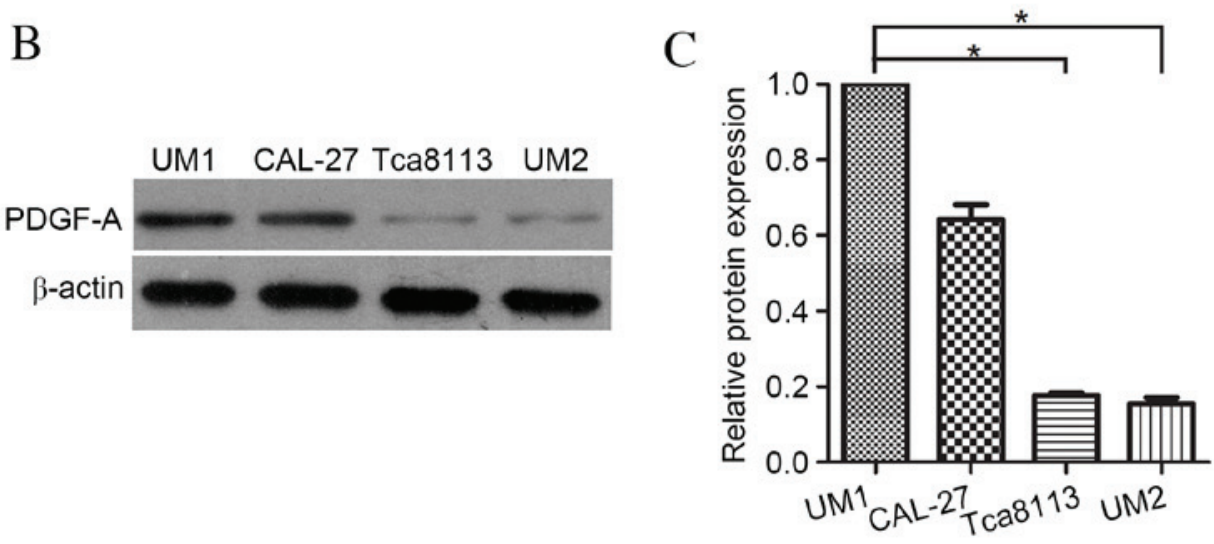

D

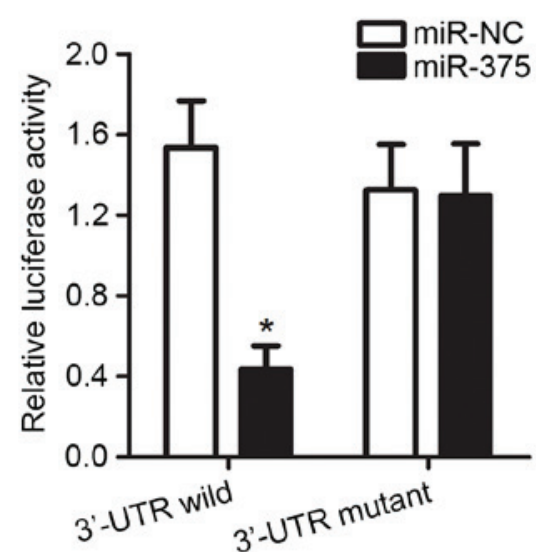

$\mathrm{E}$
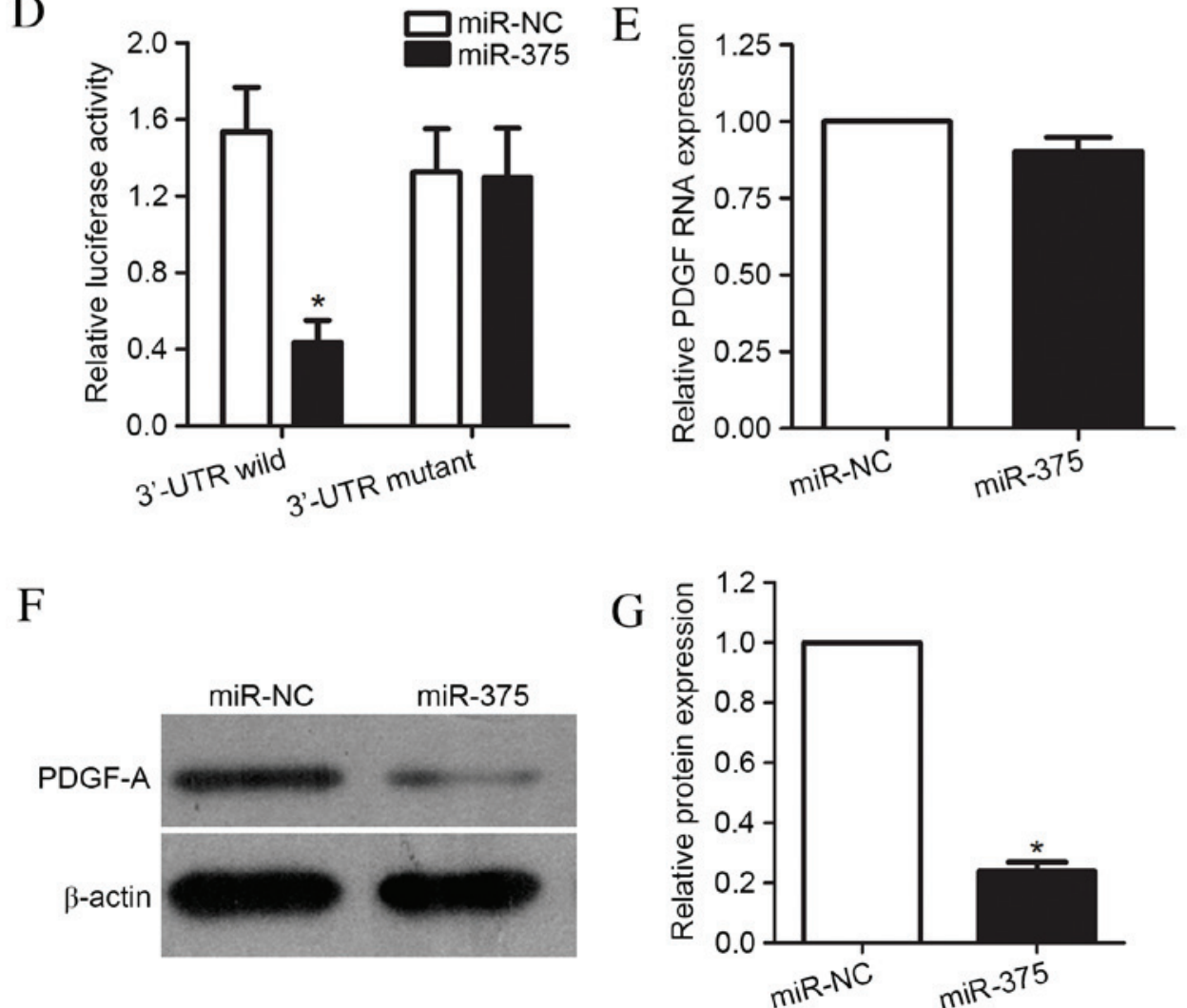

Figure 3. PDGF-A is a direct target of miR-375. (A) A predicted duplex formation between the wild-type PDGF-A 3'-UTR and miR-375. The mutant PDGF-A sequence is presented, mutated at the predicted binding site. (B) Western blot analysis demonstrated PDGF-A protein expression levels in oral squamous cell carcinoma cell lines. (C) Western blots were quantified by normalization to $\beta$-actin, which served as an internal loading control. ${ }^{*} \mathrm{P}<0.05$. (D) Luciferase activity of wild-type or mutant PDGF-A 3'-UTR-containing reporters in UM1 cells transfected with miR-375 mimic or miR-NC. "P<0.05 vs. miR-NC. (E) Reverse transcription-quantitative polymerase chain reaction analysis of PDGF-A mRNA expression levels in UM1 cells transfected with miR-375 mimic or miR-NC. Data were normalized to GAPDH mRNA. (F) Western blotting images of PDGF-A in UM1 cells transfected with miR-375 mimic or miR-NC. (G) Western blots were quantified by normalization to $\beta$-actin, which served as an internal loading control. ${ }^{*} \mathrm{P}<0.05$ vs. miR-NC. Data are presented as the mean \pm standard deviation. miR-375, microRNA-375; miR-NC, microRNA negative control; PDGF-A, platelet-derived growth factor-A; UTR, untranslated region.

with miR-375 mimic- and NC-transfected cells $(\mathrm{P}<0.001$; Fig. 4F). These results suggested that miR-375 affected the migration and invasion of UM1 cells via regulation of its target gene, PDGF-A, and that overexpression of PDGF-A was able to reverse the suppressive effect of miR-375 in UM1 cells.

\section{Discussion}

miRNAs have been reported to serve essential roles in the progression of oral cancer (14-16). Therefore, miRNAs may be promising novel targets for the development of oral cancer 
A

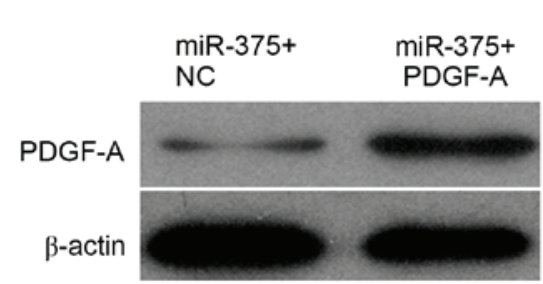

C

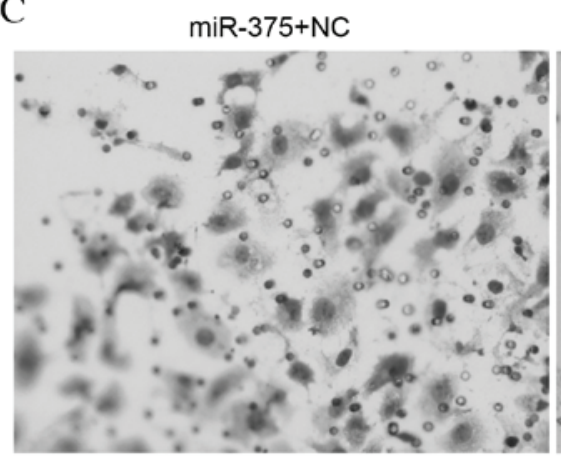

D

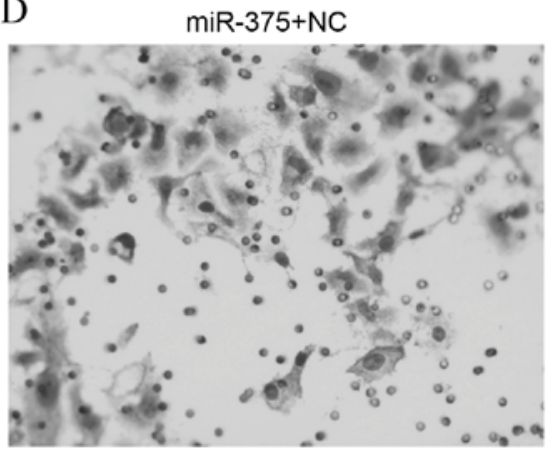

E

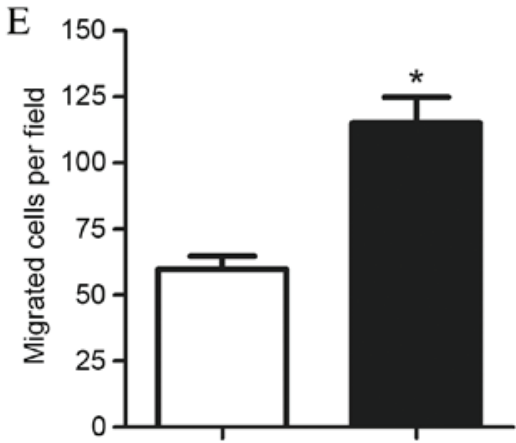

miR-375+NC $m$ miR-375+PDGF-A

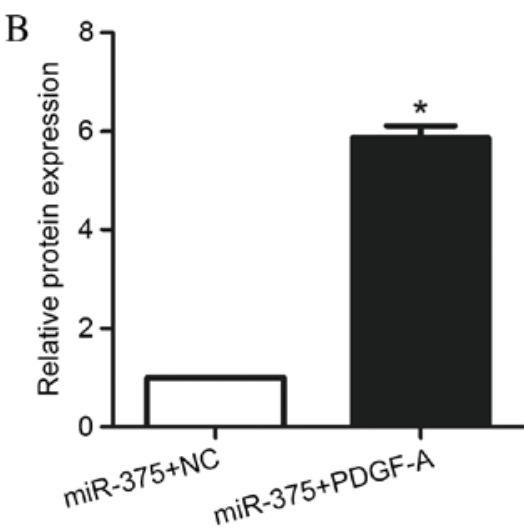

miR-375+PDGF-A
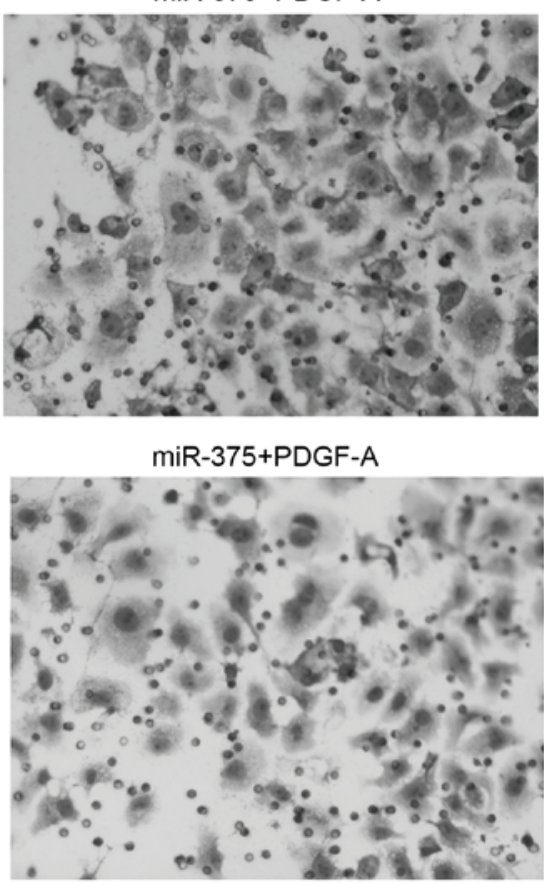

F

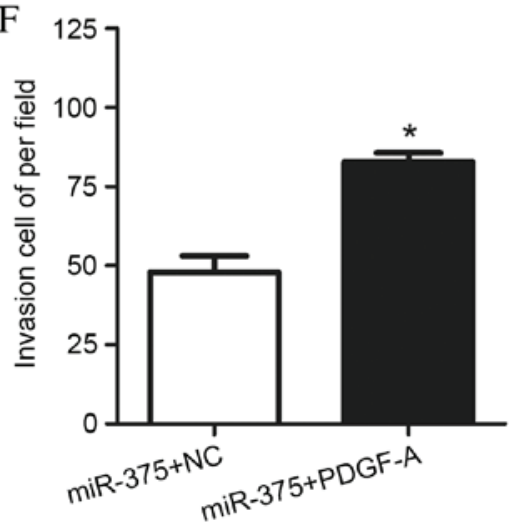

Figure 4. Effects of PDGF-A overexpression on UM1 cell migration and invasion following transfection with miR-375. (A) PDGF-A protein expression levels $48 \mathrm{~h}$ after transfection with PDGF-A or NC and miR-375 mimic were detected by western blotting. (B) Western blots were quantified by normalization to $\beta$-actin, which served as an internal loading control. (C) Representative images of UM1 cell (C) migration and (D) invasion, as detected by Transwell assays, $48 \mathrm{~h}$ after transfection with miR-375 mimic plus NC or PDGF-A. The average number of (E) migrating or (F) invading cells per field in the miR-375 plus NC and miR-375 plus PDGF-A groups. Data are presented as the mean \pm standard deviation. ${ }^{*} \mathrm{P}<0.05$ vs. miR-375 plus NC. miR-375, microRNA-375; NC, negative control; PDGF-A, platelet-derived growth factor-A.

treatments. miR-375, which is frequently downregulated in oral cancer, is a prognostic biomarker for early-stage OSCC patients (17). Shi et al (12) demonstrated that miR-375 was involved in the premalignant progression of OSCC, via interactions with the transcription factor KLF5, which modulates the expression levels of genes contributing to proliferation 
and apoptosis. The present study aimed to investigate the regulatory role of miR-375 in OSCC cell migration and invasion. The results revealed that miR-375 was downregulated in highly metastatic, compared with less metastatic, OSCC cell lines. Additionally, overexpression of miR-375 suppressed the migration and invasion of UM1 cells. These results indicated that reduced expression levels of miR-375 may induce more aggressive tumor behavior during oral cancer progression. These results are similar to those reported by Siow et al (18), who demonstrated that low miR-375 expression levels were significantly associated with late-stage disease, larger tumor size and a non-cohesive pattern of invasion in OSCC.

To investigate the underlying mechanisms by which miR-375 contributes to UM1 migration and invasion, software tools were used to predict the target genes of miR-375. PDGF-A was of particular interest due to its role in the migration and invasion of cells during cancer development. PDGF was originally isolated from blood platelets as a growth factor for cells of mesenchymal origin, but the $P D G F$ gene is additionally expressed by a variety of cell types throughout development and in adult vertebrates. PDGF-A serves a key role in the regulation of multiple biological functions, including vascularization, cell migration and invasion, and tumor development. Emerging evidence implicates PDGF-A as a potential prognostic marker, independent of the traditional pathologic parameters $(19,20)$. The promoter of this gene is a potential target for an efficient and selective antineoplastic gene therapy in multiple cancer types, including breast cancer and osteosarcoma $(21,22)$; however, the biological functions of PDGF-A in OSCC remain to be determined. Identifying the association between miR-375 and PDGF-A, which is essential for tumor progression, may provide novel therapeutic targets. As demonstrated in the present study, the protein expression levels of PDGF-A in the highly metastatic cell lines was significantly greater compared with less metastatic cell lines. The results revealed that the expression levels of PDGF-A were negatively associated with the expression levels of miR-375. Furthermore, these data indicated that miR-375 directly bound to the 3'-UTR of PDGF-A mRNA and affected PDGF-A expression levels in UM1 cells. Overexpression of PDGF-A significantly reversed the suppressive effect of miR-375 on OSCC migration and invasion. The reversal effect was full, as the migration and invasion ability of miR-375 mimic- and PDGF-A-transfected cells was similar to that of NC-transfected only cells. These results indicated that PDGF-A is a potential tumor promoter that induces increased migration and invasion in the UM1 OSCC cell line, consistent with the previously described functions of this protein in other types of cancer (22).

In conclusion, the present study demonstrated that the expression levels of miR-375 were significantly reduced in highly metastatic compared with less metastatic OSCC cell lines, and that miR-375 inhibited cell migration and invasion by targeting the tumor promoter PDGF-A in UM1 cells. Therefore, miR-375 may be a promising therapeutic target for the treatment of oral cancer.

\section{References}

1. Ferlay J, Shin HR, Bray F, Forman D, Mathers C and Parkin DM: Estimates of worldwide burden of cancer in 2008: GLOBOCAN 2008. Int J Cancer 127: 2893-2917, 2010.
2. Gupta S, Kong W, Peng Y, Miao Q and Mackillop WJ: Temporal trends in the incidence and survival of cancers of the upper aerodigestive tract in Ontario and the United States. Int J Cancer 125: 2159-2165, 2009

3. Wikner J, Gröbe A, Pantel K and Riethdorf S: Squamous cell carcinoma of the oral cavity and circulating tumour cells. World J Clin Oncol 5: 114-124, 2014.

4. Bartel DP and Chen CZ: Micromanagers of gene expression: The potentially widespread influence of metazoan microRNAs. Nat Rev Genet 5: 396-400, 2004.

5. Bartel DP: MicroRNAs: Genomics, biogenesis, mechanism, and function. Cell 116: 281-297, 2004.

6. Hwang HW and Mendell JT: MicroRNAs in cell proliferation, cell death and tumorigenesis. Br J Cancer 94: 776-780, 2006.

7. Winther M, Alsner J, Tramm T, Baeksgaard L, Holtved E and Nordsmark M: Evaluation of miR-21 and miR-375 as prognostic biomarkers in esophageal cancer. Acta Oncol 54: 1582-1591, 2015.

8. Hu A, Huang JJ, Xu WH, Jin XJ, Li JP, Tang YJ, Huang XF, Cui HJ, Sun GB, Li RL, et al: miR-21/miR-375 ratio is an independent prognostic factor in patients with laryngeal squamous cell carcinoma. Am J Cancer Res 5: 1775-1785, 2015.

9. Mao Q, Quan T, Luo B, Guo X, Liu L and Zheng Q: miR-375 targets KLF4 and impacts the proliferation of colorectal carcinoma. Tumour Biol 37: 463-471, 2016.

10. Jia L, Huang Y, Zheng Y, Lyu M, Zhang C, Meng Z, Gan Y and Yu G: miR-375 inhibits cell growth and correlates with clinical outcomes in tongue squamous cell carcinoma. Oncol Rep 33: 2061-2071, 2015

11. Song L, Liu S, Zeng S, Zhang L and Li X: miR-375 modulates radiosensitivity of HR-HPV-positive cervical cancer cells by targeting UBE3A through the p53 pathway. Med Sci Monit 21: 2210-2217, 2015

12. Shi W, Yang J, Li S, Shan X, Liu X, Hua H, Zhao C, Feng Z, Cai Z, Zhang L, et al: Potential involvement of miR-375 in the premalignant progression of oral squamous cell carcinoma mediated via transcription factor KLF5. Oncotarget 6: 40172-40185, 2015.

13. Livak KJ and Schmittgen TD: Analysis of relative gene expression data using real-time quantitative PCR and the 2(-Delta Delta C(T)) Method. Methods 25: 402-408, 2001.

14. Yu X and Li Z: MicroRNA expression and its implications for diagnosis and therapy of tongue squamous cell carcinoma. J Cell Mol Med 20: 10-60, 2016.

15. Xu H, Yang Y, Zhao H, Yang X, Luo Y, Ren Y, Liu W and Li N: Serum miR-483-5p: A novel diagnostic and prognostic biomarker for patients with oral squamous cell carcinoma. Tumour Biol 37: 447-453, 2016.

16. Saad MA, Kuo SZ, Rahimy E, Zou AE, Korrapati A, Rahimy M, Kim E, Zheng H, Yu MA, Wang-Rodriguez J and Ongkeko WM: Alcohol-dysregulated miR-30a and miR-934 in head and neck squamous cell carcinoma. Mol Cancer 14: 181, 2015.

17. Yoon AJ, Wang S, Shen J, Robine N, Philipone E, Oster MW, Nam A and Santella RM: Prognostic value of miR-375 and miR-214-3p in early stage oral squamous cell carcinoma. Am J Transl Res 6: 580-592, 2014.

18. Siow MY, Ng LP, Vincent-Chong VK, Jamaludin M, Abraham MT, Abdul Rahman ZA, Kallarakkal TG, Yang YH, Cheong SC and Zain RB: Dysregulation of miR-31 and miR-375 expression is associated with clinical outcomes in oral carcinoma. Oral Dis 20: 345-351, 2014.

19. Katano M, Nakamura M, Fujimoto K, Miyazaki K and Morisaki T: Prognostic value of platelet-derived growth factor-A (PDGF-A) in gastric carcinoma. Ann Surg 227: 365-371, 1998.

20. Jones AC, Antillon KS, Jenkins SM, Janos SN, Overton HN, Shoshan DS, Fischer EG, Trujillo KA and Bisoffi M: Prostate field cancerization: Deregulated expression of macrophage inhibitory cytokine 1 (MIC-1) and platelet derived growth factor A (PDGF-A) in tumor adjacent tissue. PLoS One 10: $\mathrm{e} 0119314,2015$

21. Mishra A, Ormerod AK, Cibull ML, Spear BT, Kraner SD and Kaetzel DM: PDGF-A promoter and enhancer elements provide efficient and selective antineoplastic gene therapy in multiple cancer types. Cancer Gene Ther 16: 298-309, 2009.

22. Yu G, Zhou A, Xue J, Huang C, Zhang X, Kang SH, Chiu WT, Tan C, Xie K, Wang J and Huang S: FoxM1 promotes breast tumorigenesis by activating PDGF-A and forming a positive feedback loop with the PDGF/AKT signaling pathway. Oncotarget 6 : 11281-11294, 2015. 